\title{
Dark Matter and Baryons (Surplus Quarks) Generated by Oblique Confinement of Quarks
}

\author{
Leif Matsson \\ Department of Physics, University of Gothenburg, Gothenburg, Sweden \\ Email:leif.matsson@telia.com
}

How to cite this paper: Matsson, L. (2020) Dark Matter and Surplus Quarks (for Baryons) Generated by Oblique Confinement of Quarks. Journal of High Energy Physics, Gravitation and Cosmology, 6, 123-132. https://doi.org/10.4236/jhepgc.2020.61011

Received: August 7, 2019

Accepted: January 17, 2020

Published: January 20, 2020

Copyright $\odot 2020$ by author(s) and Scientific Research Publishing Inc. This work is licensed under the Creative Commons Attribution International License (CC BY 4.0).

http://creativecommons.org/licenses/by/4.0/ (c) (i) Open Access

\begin{abstract}
For surplus quarks (and baryons) to emerge after Big Bang, a nonequilibrium binding and superconductor-like condensation of quark-antiquark pairs must occur before the electroweak (EW) symmetry breakdown (similar for leptons). The formerly unknown dimensionless coupling to the Ginsburg-Landau like potential and the scale parameter in the EW theory then become microscopic functions of the massive quark and antiquark fields, thus defining the matter-antimatter asymmetry and the dark matter content in the Universe at correct orders of magnitude. Thereby also the number of free parameters in the Standard Model is reduced.
\end{abstract}

\section{Keywords}

Quark Confinement, Matter-Antimatter Asymmetry, Dark Matter, Black Holes, New Vacuum, Baryogenesis, Inflation, WIMPs

\section{Introduction}

As shown by Nielsen and Olesen [1], it is easy to construct classical field theories that allow for vortex line solutions with equations of motion equal to those of the Nambu dual string [2]. In brief, they wanted to find a link between the spectrum of the Veneziano model and local field theory in analogy with type II superconductors. 't Hooft [3] and Ezawa and Iwazaki [4] then showed that the QCD vacuum works like a dual type II superconductor with the quark-antiquark $(q \bar{q})$ pair playing the role of a Cooper pair [5]. The preparation of the QCD vacuum can thus no longer be regarded as just a filling of negative energy states because in addition, quarks must also be confined such that surplus quarks and baryons [6] can emerge after Big Bang. Infinite amounts of free massless quarks and antiquarks were then supposedly equally abundant, which corresponds to a totally empty vacuum with all negative energy states (holes) unfilled, making quantum 
field operators meaningless. A model for transition between the empty and non-empty vacuum states is therefore postulated in terms of classical fields.

Short distance behavior in QCD is usually associated with asymptotic freedom. But since all particles created at Big Bang were massless, infinite amounts of asymptotically free quark-antiquark pair components must then have filled each volume element of the Universe, implying that large numbers of such components from different pairs could have come sufficiently close within a bag-like distance and become strongly bound at zero momentum transfer. High and low energy phenomena could thus have occurred rather independently.

As will be shown, for surplus quarks and baryons to emerge, the negative energy states must be filled in a nonequilibrium manner. This is here formulated as a rate equation for binding of massless quarks to massless antiquarks (holes)

$$
\frac{\mathrm{d} \psi_{i}}{\mathrm{~d} t}=k \cdot q_{i}(\boldsymbol{x}, t) \cdot \bar{q}_{i}(\boldsymbol{x}, t)-k^{\prime} \cdot \psi_{i}(\boldsymbol{x}, t)
$$

where $q_{i}, \bar{q}_{i}$ and $\psi_{i}(\boldsymbol{x}, t)$ are classical fields, which here play the role as densities of massless quarks, antiquarks and $q \bar{q}$-pairs of flavor $i$, and $k$ and $k$ are temperature dependent association and dissociation constants. The binding of a $q \bar{q}$-pair in Equation (1a) is approximately described by a point-like bag [4], and the flavor index $i$ is henceforth suppressed. The colour index was dropped from start because quarks of all colours give the same form of result too.

By insertion of constraints, $q(\boldsymbol{x}, t)=q_{0}-\psi(\boldsymbol{x}, t)$ and $\bar{q}(\boldsymbol{x}, t)=\bar{q}_{0}-\psi(\boldsymbol{x}, t)$, where $q_{0}$ and $\bar{q}_{0}$ are the initial quark and antiquark fields, and with $a=\left(q_{0}+\bar{q}_{0}+K\right) / 2, \quad b^{2}=q_{0} \bar{q}_{0}$ and $K=k^{\prime} / k$, Equation (1a) becomes

$$
\frac{\mathrm{d} \psi}{\mathrm{d} t}=k\left((a-\psi(\boldsymbol{x}, t))^{2}-\left(a^{2}-b^{2}\right)\right)
$$

After integration, Equation (1b) yields

$$
\ln \left(\frac{a+\sqrt{a^{2}-b^{2}}-\psi}{a-\sqrt{a^{2}-b^{2}}-\psi} \cdot \frac{a(1-g)}{a(1+g)}\right)=\ln \left(\frac{q^{\prime}(\boldsymbol{x}, t)}{\bar{q}^{\prime}(\boldsymbol{x}, t)} \cdot \frac{\bar{q}_{K}}{q_{K}}\right)=2 \mathrm{kag} \cdot\left(t-t_{0}\right)
$$

where $q^{\prime}(\boldsymbol{x}, t)=q_{K}-\psi(\boldsymbol{x}, t)$ and $\bar{q}^{\prime}(\boldsymbol{x}, t)=\bar{q}_{K}-\psi(\boldsymbol{x}, t)$ are the time dependent quark and antiquark fields, $q_{K}=a(1+g) \geq q_{0}$ and $\bar{q}_{K}=a(1-g) \leq \bar{q}_{0}$ become the new "initial" fields, $g=\sqrt{\left(a^{2}-b^{2}\right) / a^{2}}=\left(q_{K}-\bar{q}_{K}\right) /\left(q_{K}+\bar{q}_{K}\right)$ and $a=\left(q_{K}+\bar{q}_{K}\right) / 2$. As will be explained, with Equation (1b) as driving equation for the vacuum dynamics, massive surplus quarks and baryons can emerge, and a matter-antimatter asymmetry and a dark matter content at correct orders of magnitude [7] be obtained.

By contrast, in lattice QCD the quark-antiquark ratio is limited by the partition function to fluctuations about one and the same initial value. Under such conditions, the Universe would have been left in a symmetric state without surplus quarks containing just $q \bar{q}$-pairs, $[q \bar{q}][q \bar{q}][q \bar{q}] \cdots$. This was one of all problems we confronted in a previous attempt to study the quark-antiquark binding [8]. In this nonequilibrium model, however, $\left(q_{K}-\bar{q}_{K}\right)$ has increased from zero, implying that surplus quarks have emerged. This can be described as 
a rearranged $q \bar{q}$-pairing, $q[\bar{q} q][\bar{q} q][\bar{q} q] \cdots$, that took place when the temperature decreased after Big Bang. To see that no antiquarks were left behind without quark partners, the quarks $q_{K}$ are divided into two sets, the surplus quarks $q_{S}$ and the non-surplus quarks $q_{N S}$. Accordingly, $\left(q_{K}-\bar{q}_{K}\right)=\left(q_{S}+q_{N S}-\bar{q}_{K}\right)=q_{S}$, because $q_{N S}=\bar{q}_{K}$. Apart from the surplus quarks $q_{S}$ which were thus "frozen out" from vacuum, the denominator of $g=q_{S} /\left(q_{S}+q_{N S}+\bar{q}_{K}\right)$ then contains just the equal numbers of quarks and antiquarks, $q_{N S}$ and $\bar{q}_{K}$. As will be further explained here, a similar reasoning should hold for leptons.

\section{Condensation}

The condensation of $q \bar{q}$-pairs can be approximately described by a geometric series

$$
\varphi(\boldsymbol{x}, t)=\sqrt{a^{2}-b^{2}}\left(1+\sum_{\eta=1}^{\infty}[\psi(\boldsymbol{x}, t) / a]^{\eta}\right)=\frac{\sqrt{a^{2}-b^{2}}}{1-\psi(\boldsymbol{x}, t) / a},
$$

which can also be interpreted as a "partition" function, where $\psi(\boldsymbol{x}, t)$ plays the role of nonequilibrium "fugacity". Equation (3) combined with Equation (1b) then yields

$$
\frac{\mathrm{d} \varphi}{\mathrm{d} t}=k g\left(a^{2}-\varphi(\boldsymbol{x}, t)^{2}\right),
$$

with solution

$$
\varphi(t)=a \tanh (\text { kagt }) .
$$

When $\varphi(t)$ is interpreted as a travelling wave with $k$ as the traveling wave velocity, Equation (4) becomes

$$
\frac{\mathrm{d} \varphi}{\mathrm{d} x}=g\left(a^{2}-\varphi(\boldsymbol{x}, t)^{2}\right)
$$

which yields a microscopic form of the Ginsburg-Landau (GL) like potential $V(\varphi)$ in the EW theory

$$
\left(\frac{\mathrm{d} \varphi}{\mathrm{d} x}\right)^{2}=\lambda\left(a^{2}-\varphi(\boldsymbol{x}, t)^{2}\right)^{2} \equiv V(\varphi)
$$

The formerly unknown coupling $\lambda=\left(q_{K}-\bar{q}_{K}\right)^{2} /\left(q_{K}+\bar{q}_{K}\right)^{2}$ and the scale parameter $a=\left(q_{0}+\bar{q}_{K}\right) / 2$ have now become microscopic functions of the massive quark and antiquark fields. However, in quantum field theory, the density of surplus quarks is defined by the square of the surplus quark field $\left(q_{K}-\bar{q}_{K}\right)^{2}$. The EW symmetry breakdown, $\varphi \rightarrow-a+\varphi$, yields the equation of motion

$$
\frac{\mathrm{d}^{2} \varphi}{\mathrm{d} t^{2}}-\frac{\mathrm{d}^{2} \varphi}{\mathrm{d} x^{2}}+(2 a g)^{2} \varphi=6 g^{2} \varphi^{2}\left(a-\frac{\varphi}{3}\right)
$$

where $2 a g$ plays the role of Higgs boson mass $m_{H}$, which equals approximately the top quark mass $m_{\text {top }} \approx q_{S}=\left(q_{K}-\bar{q}_{K}\right)=2 a g=m_{H}$. Although this is better than the estimate $m_{H} \approx 2 m_{\text {top }}$ derived from a gauged Nambu-Jona-Lasino me- 
chanism [9], it is still not acceptable. Because the expression for surplus quarks $q_{S}=\left(q_{K}-\bar{q}_{K}\right)$ was derived for massless quarks, and a small error in the approximation $m_{\text {top }} \approx q_{S}=m_{H}$, could then grow with $a$ as in Equation (5).

However, this flaw can be remedied by modifying the normalization of the condensation field, $\varphi \rightarrow \xi \varphi \equiv \varphi_{\xi}$. Equation (6) then becomes

$$
\frac{\mathrm{d} \varphi_{\xi}}{\mathrm{d} x}=g\left(a^{2} \xi^{2}-\varphi_{\xi}^{2}\right) \text {, }
$$

provided that $k \rightarrow k / \xi$. The relationship $2 a g=q_{S} \approx m_{\text {top }}$ still holds approximately, but the Higgs boson mass is now defined as $m_{H}=2 a g \xi$, where $\xi=m_{H} / m_{\text {top }}<1$. The reduction of the condensation field by a factor $\xi$, which corresponds to an increase of the rate constant, $k \rightarrow k / \xi$, was required to obtain a lowering of the Higgs boson mass $m_{H}=2 a g \xi$ compared to $m_{\text {top }}$. This could also be interpreted as a metric extension of space, $x=t \cdot k \rightarrow x=t \cdot k / \xi$. The condensation field $\varphi_{\xi}$ thus becomes linked to the rapid cosmic inflation that took place between $10^{-36}-10^{-32} \mathrm{~s}$ after Big Bang. Thereafter a slower rate of inflation is expected.

The process can also be followed backwards in time until Big Bang, at which all surplus quarks $\left(q_{K}-\bar{q}_{K}\right)=2 a g$, the coupling $\lambda=g^{2}=\left(q_{K}-\bar{q}_{K}\right)^{2} /\left(q_{K}+\bar{q}_{K}\right)^{2}$, and all masses vanish together with the mass of the Higgs boson, which in this model no longer plays the role of an elementary particle. It essentially consists of $q \bar{q}$-pairs, predominantly a massive top $q \bar{q}$-pair, which thus plays a more fundamental intermediate role in the generation of mass than the Higgs boson. However, all particle masses are now due to binding energy [10].

As mentioned before here, a superconductor like model for confinement of quarks is not a new idea [1] [2] [3] [4] [5] [9] [11] [12]. But for massive surplus quarks and baryons to emerge after Big Bang, quarks had to be confined by a nonequilibrium dynamics, as in Equation (1b), Equation (3) and Equation (7). With a totally empty vacuum at Big Bang, the Standard Model (SM) breaks down together with the quantum fields. The connection between high energy particle physics and cosmology thus goes beyond the SM, and obviously also beyond the Fermi theory.

The emergence of surplus leptons after Big Bang can be described by the nonequilibrium dynamics defined by Equation (1b), Equation (3) and Equation (7), provided that the bags can be replaced by a contact interaction like in the Fermi model, however, with leptons described by classical fields. Even in this case Equation (1a) and Equation (1b) can describe the emergence of all massless surplus leptons, because neutrino oscillations start only when neutrinos begin to acquire mass.

\section{Matter-Antimatter Asymmetry and Dark Matter}

The coupling $\lambda=g^{2}$ to the GL potential in the EW theory, a hitherto unknown parameter in the SM, has here become an asymmetric function of the 
massive quark and antiquark fields,

$$
g=\frac{q_{K}-\bar{q}_{K}}{q_{K}+\bar{q}_{K}}=\frac{q_{S}}{q_{S}+q_{N S}+\bar{q}_{K}} .
$$

Regardless of which quark actually contributes, $\lambda$ could thus give an estimate of the matter-antimatter asymmetry in the Universe. Moreover, since the emergence of surplus quarks $q_{s}$ is a prerequisite for the emergence of nucleons and ordinary matter, $\lambda=g^{2}=q_{S}^{2} /\left(q_{S}+q_{N S}+\bar{q}_{K}\right)^{2} \quad$ could also become a key to discriminate between ordinary and non-ordinary matter, and hence a possible tool to identify dark matter. With $\xi=m_{H} / m_{\text {top }}=1$ and $a \approx 173 \mathrm{GeV}$, a Higgs boson mass $m_{H}=2 g a \approx 125 \mathrm{GeV}$ would have yielded $g \approx 0.36$, and a matter to all matter ratio $\lambda=g^{2} \approx 0.13$, not far from the 0.15 ( $g=0.39$ ) observed [7]. By contrast to all heavier material, which should have decayed, the contribution from nucleons could then make $\lambda$ increase towards the 0.15 observed. This is expected to occur at the chiral transition, after which a more sustainable form of ordinary matter could emerge from protons and neutrons. But already with a partially refilled vacuum, quantum effects start to contribute and hence masses must be renormalized, implying that the hitherto used top quark mass is just an intermediate mass. Since there are no bare masses to start from, this intermediate mass is interpreted as a running top quark mass $m_{\text {rtop }}$, which like $m_{\text {top }}$ is expressed here in units of the Higgs boson mass $m_{H}$. The problem is thus reduced to obtaining the mass renormalization of $m_{\text {rtop }}$.

For simplicity, the results for light quarks derived in a quenched lattice QCD are used. The renormalization group invariant top quark mass $m_{\text {top }}$ to the running quark mass $m_{\text {rtop }}$ ratio is estimated to $m_{\text {top }} / m_{\text {rtop }}=1.157$ [13]. Thus, if the running top quark mass $m_{\text {rtop }}$ equals the assessed top quark mass $173.1 \mathrm{GeV}$ divided by 1.157 , which gives $m_{\text {rtop }}=149.6 \mathrm{GeV}$, then $\xi=m_{H} / m_{\text {rtop }}=125 / 149.6$.

Since $m_{H}=2 a g \xi=q_{S} \xi=125 \mathrm{GeV}$, the coupling $g$ can be written as

$$
g=\frac{\xi q_{S}}{\xi q_{S}+\xi\left(q_{N S}+\bar{q}_{K}\right)}=\frac{m_{H}}{m_{H}+\xi\left(q_{N S}+\bar{q}_{K}\right)}=0.36
$$

implying that the free dark matter components $q_{N S}$ and $\bar{q}_{K}$ must have formed a dark bound state at about $\xi\left(q_{N S}+\bar{q}_{K}\right)=222 \mathrm{GeV}$ (WIMPs?). But like the Higgs boson, this bound state is expected to have decayed into lighter material and dark energy. With a total maximal mass of $346 \mathrm{GeV}$, the two top quark components $q_{K}$ and $\bar{q}_{K}$ also seem to have been the most likely candidates for initiation of black holes and dark matter halos.

At temperatures below $1 \mathrm{GeV}$, all heavy material, observable as well as dark, should have decayed into lighter material plus dark energy, and the dark energy density $\rho_{\Lambda}$ should have increased correspondingly. Provided that the bag like interaction in Equation (1a) can be replaced by a Fermi like weak contact interaction, Equation (10) and Equation (11) should work also for leptons.

After the condensation process, which was here synchronized with the cosmic inflation, the matter and dark matter densities are expected to have changed 
more slowly. However, if $\Omega_{M}+\Omega_{D M}$ (in units of the critical density) had increased from zero at Big Bang to just $0.317\left(\Omega_{M}=0.049\right.$ plus $\Omega_{D M}=0.268$ ) at the EW transition, hence with $\Omega_{\Lambda}=0.683$, the Universe would not have been matter dominated until about 4 billion years ago [14], at which $\Omega_{M}+\Omega_{D M}$ was equal to $\Omega_{\Lambda} \approx 0.5$, and after which the dark energy began to dominate. For simplicity, it is here assumed that $\Omega_{M}+\Omega_{D M}$ has decreased linearly after Big Bang to the 0.317 observed today. This implies that the total mass $\Omega_{M}+\Omega_{D M}$ should have increased to 0.948 at the EW transition, hence with $\Omega_{\Lambda}=0.052$, $\Omega_{D M}=0.825$ and $\Omega_{M}=0.13 \cdot\left(\Omega_{M}+\Omega_{D M}\right)=0.123$.

Immediately after Big Bang, when $\Omega_{M} \sim 10^{-9}, \lambda \sim 10^{-9}$ and $\Omega_{\Lambda} \sim 10^{-9}$, the remaining $\Omega_{D M} \sim 1-2 \times 10^{-9}$ could have been identified as "dark matter". But since mass was then not yet generated, the sum $\Omega_{M}+\Omega_{D M} \sim 1-1 \times 10^{-9}$ must be identified as energy, and the vacuum energy released at Big Bang must have been $\Omega_{\Lambda} \sim 1$.

At the chiral transition, the sigma meson is assumed to have played the role of Higgs boson [9] [15]. Earlier decays of heavy quark-antiquark material should then have implied a decrease of ordinary and dark matter contents, and due to more stable nucleon contributions, the coupling should now have increased to $\lambda \approx 0.15$ ( $g=0.39$ ). A sigma mass at about $600 \mathrm{MeV}$ would then give a dark bound state at about $938 \mathrm{MeV}, g=0.39 \approx 600 /(600+938)$, a more stable WIMP candidate. Electrons, muons, tau leptons and their antileptons are also expected to have contributed to the observed $\lambda=0.15$.

The SM prediction that neutrinos were massless was disproved by the neutrino oscillation experiments [16], which showed that also neutrinos have mass and that the neutrino flavour eigenstates do not coincide with the neutrino mass eigenstates. But since the lepton-antilepton asymmetry, like the quark-antiquark asymmetry in $g$, emerged before mass, and since mass enters equally and simultaneously for all neutrinos and antineutrinos, Equation (10) and Equation (11) should hold also in this case. Given that all heavier dark bound states have decayed and contributed to dark energy, and that neutrinos have the lowest masses, $\lambda$ should finally have attained the $0.15(g=0.39)$ observed. Insertion of the tau-neutrino mass $18.2 \mathrm{MeV}$ then gives $g=18.2 /(18.2+28.47)=0.39$ and a dark bound state at $28.47 \mathrm{MeV}$. The muon- and electron-neutrino masses, 0.17 $\mathrm{MeV}$ and $1.0 \mathrm{eV}$, similarly give dark bound states at $0.27 \mathrm{MeV}$ and $1.56 \mathrm{eV}$.

\section{Discussion}

The suggested model yields a form of oblique quark confinement, by which surplus quarks emerge, and where magnetic monopoles play a hidden role in the confinement process [1] [3] [4] [5]. The numbers of particles and antiparticles were here assumed to be equal at Big Bang, and thus vacuum must have been totally empty. With a totally empty vacuum, however, the definitions of quantum fields and the SM were lost. The construction of the model thus had to start with a filling of vacuum in terms of classical fields. 
To describe the increase of surplus quarks and leptons relative to their antiparticles, the negative energy states (the holes) in the empty vacuum, had to be filled in a nonequilibrium manner. Because a thought filling of vacuum "by hand", as Dirac did it, would have given a constant particle/antiparticle ratio already from start. The filling of the empty vacuum states was here described as a bag-like binding of quarks to antiquarks. Then, after condensation of the strongly bound quark-antiquark pairs, a superconductor like interaction emerged in which the quark-antiquark pairs played the role as Cooper pairs.

The nonequilibrium vacuum dynamics, which here defined the nonperturbative infrared slavery part of QCD, generated the GL like potential of the EW theory, a form of partial grand unification of the actual forces. Except for the microscopic dependence of the coupling and scale parameters on the massive quark and antiquark variables, and that the Higgs boson does not play a role as elementary particle here, the Standard Model should work as before. The suggested model could hopefully also describe how surplus leptons emerge. How surplus massless neutrinos acquire mass can then be described as usual [16] [17].

The formerly unknown coupling $\lambda=g^{2}$ to the GL potential has here become known as an asymmetric function of the quark and antiquark variables, thereby reducing the number of free parameters in the SM and providing a possible tool to determine the matter-antimatter asymmetry and the dark matter content in the Universe. By following the increase of the matter to all matter ratios and the decrease of dark matter after Big Bang, also the increase of dark energy, i.e. of the cosmological constant [18], could be followed.

In the case of neutrinos, however, the problem is more complicated, because the neutrino flavour eigenstates do not coincide with the neutrino mass eigenstates [16] [17]. But since Equation (9) is independent of flavor and mass, $\lambda$ could still yield the correct matter to all matter ratios for each kind of neutrino, at different stages after Big Bang. Thus, after acquiring mass, also the neutrino-antineutrino bound states are expected to have contributed to the ordinary and dark masses, and after the decay of all heavier dark matter, the coupling to the GL potential should then finally have reached the $\lambda=0.15$ observed. Since also the parts of masses acquired via the Higgs boson are due to binding energy between particles and antiparticles, the entire masses of all particles should be due to binding energy as hinted already by Wilczek [10].

The suggested model describes the emergence of surplus quarks in a Big Bang Universe containing infinite and equal numbers $(n=\infty)$ of massless $(m=0)$ quarks and antiquarks per unit volume. How gravitation enters together with mass into the model can then be understood from the formula for conservation of energy density, $\rho_{0} c^{2}=0 \cdot \infty c^{2} \rightarrow m_{0} n_{0} c^{2}$, where $n_{0}$ is the number of surplus quarks with mass $m_{0}$ per volume unit in a cloud of surplus quarks at rest. Seen from a moving frame at velocity $v=\beta c$ the quarks become more massive and the volume element with a fixed number of quarks becomes Lorentz contracted. This implies that $\rho_{0} \rightarrow \rho=\rho_{0} \gamma^{2}$, where $\gamma^{2}=1 /\left(1-\beta^{2}\right)$, and $\rho c^{2}$ thus behaves 
like a second rank tensor. Viewed from the moving frame, $\rho_{0} c^{2}$ thus behaves like the stress-energy tensor $T^{\mu v}=\rho_{0} v^{\mu} v^{v}$, the source of gravitation in the theory of general relativity. The actual model should thus also be in compliance with the observation of gravitational waves [19] [20]. Soon after Big Bang the top quarks and antiquarks, seem to have been the dominating ordinary and dark mass sources of gravitation, but it is clearly also a question of abundance. Like mass, also gravitation must have been chiefly generated by the gluonic field interactions.

It could be speculated that the dark masses become subjected to the strongest gravitational forces towards the centre of black holes, at which the mass-energy density should increase correspondingly. The dark $q \bar{q}$-pairs should then be heated up and disintegrate into infinite amounts of massless dark $q \bar{q}$-pair components (similar for leptons), because our model should then work backwards. Such processes could also be responsible for launching of jets from black holes, and the jet particles could then become massive again as described here.

A preliminary version of the model has been presented earlier [21] [22]. Also, this version can be further modified e.g. by letting dark matter start dominating earlier or later than 4 billion years ago. The sum $\Omega_{M}+\Omega_{D M}$ should have a different nonlinear behaviour in time depending on if dark matter or dark energy dominates. Since top quarks are not light, and unquenched effects are expected to contribute, the renormalization group invariant top quark mass to running top quark mass ratio probably deviates from 1.157 [13]. The sigma meson mass is not exactly $600 \mathrm{MeV}$. Black hole conditions could have delayed the decay of heavier materials, to mention just some. More data are obviously needed to optimize this puzzle and to test the model [7] [16] [23] [24] [25] [26]. As Peebles and Ratra put it [18], "It is best to wait and see what the physics of baryogenesis and neutrinos teach us". Hopefully, this model could then fill in some gaps.

\section{Acknowledgements}

I am grateful to Ludvig Faddeev, whose memory I wish to honor, for discussions on these items at CERN and Stecklow Mathematical Institute. I also thank Holger Nielsen for more recent useful discussions at Niels Bohr Institute and for a critical reading of the manuscript.

\section{Conflicts of Interest}

The author declares no conflicts of interest regarding the publication of this paper.

\section{References}

[1] Nielsen, H.B. and Olesen, P. (1973) Vortex-Line Models for Dual Strings. Nuclear Physics B, 61, 45-61. https://doi.org/10.1016/0550-3213(73)90350-7

[2] Nambu, Y. (1974) Strings, Monopoles, and Gauge Fields. Physical Review D, 10, 4262-4268. https://doi.org/10.1103/PhysRevD.10.4262 
[3] 't Hooft, G. (1981) Topology of the Gauge Condition and New Confinement Phases in Non-Abelian Gauge Theories. Nuclear Physics B, 190, 455-478. https://doi.org/10.1016/0550-3213(81)90442-9

[4] Ezawa, Z.F. and Iwazaki, A. (1982) Abelian Dominance and Quark Confinement in Yang-Mills Theories. Physical Review D, 25, 2681-2689. https://doi.org/10.1103/PhysRevD.25.2681

[5] Kondo, K.-I. (1998) Abelian-Projected Effective Gauge Theory of QCD with Asymptotic Freedom and Quark Confinement. Progress of Theoretical Physics Supplements, 131, 243-255. https://doi.org/10.1143/PTPS.131.243

[6] Sakharov, A. (1967) Violation of CP Invariance, C Asymmetry, and Baryon Asymmetry of the Universe. JETP Letters, 5, 24-27.

[7] NASA News (2013) Planck Mission Brings Universe Into Shap Focus. https://www.astronomy.com/news71013/03/

[8] Matsson, L. and Meuldermans, R. (1977) Long Range Correlations in Forward Quark(anti-) Quark Scattering in QCD. Physics Letters B, 70, 309-312.

https://doi.org/10.1016/0370-2693(77)90665-7

[9] Bardeen, W.A., Hill, C.T. and Lindner, M. (1990) Minimal Dynamical Symmetry Breaking of the Standard Model. Physical Review D, 41, 1647-1660. https://doi.org/10.1103/PhysRevD.41.1647

[10] Wilczek, F. (2012) Origins of Mass. Central European Journal of Physics, 10, 1021-1037. https://doi.org/10.2478/s11534-012-0121-0

[11] Nambu, Y. and Jona-Lasino, G. (1961) Dynamical Model of Elementary Particles Based on an Analogy with Superconductivity. I. Physical Review, 121, 345-358. https://doi.org/10.1103/PhysRev.122.345

[12] Mandelstam, S. (1976) II. Vortices and Quark Confinement in Non-Abelian Gauge Theories. Physics Reports, 23, 245-249. https://doi.org/10.1016/0370-1573(76)90043-0

[13] Capitani, S., Lüscher, M., Sommer, R. and Wittig, H. (1999) Non-Perturbative Quark Mass Renormalization in Quenched Lattice QCD. Nuclear Physics B, 544, 669-698. https://doi.org/10.1016/S0550-3213(98)00857-8

[14] Frieman, J.A., Turner, M.S. and Huterer, D. (2008) Dark Energy and the Accelerating Universe. Annual Review of Astronomy and Astrophysics, 46, 385-432. https://doi.org/10.1146/annurev.astro.46.060407.145243

[15] Mishustin, I.N. and Scavenius, O. (1997) Dynamical Generation of the Constituent Mass in Expanding Plasma. Physics Letters B, 396, 33-38. https://doi.org/10.1016/S0370-2693(97)00136-6

[16] Fukuda, Y., et al. (1998) Evidence for Oscillation of Atmospheric Neutrinos. Physical Review Letters, 81, 1562-1567. https://doi.org/10.1103/PhysRevLett.81.1562

[17] Di Bari, P. (2012) An Introduction to Leptogenesis and Neutrino Properties. Contemporary Physics, 53, 315-338. https://doi.org/10.1080/00107514.2012.701096

[18] Peebles, P.J.E. and Ratra, B. (2003) The Cosmological Constant and Dark Energy. Reviews of Modern Physics, 75, 559-606. https://doi.org/10.1103/RevModPhys.75.559

[19] Abbott, B.P., et al. (2016) Observation of Gravitational Waves from a Binary Black Hole Merger. Physical Review Letters, 116, Article ID: 061102.

[20] Corda, C. (2009) Interferometric Detection of Gravitational Waves: The Definitive Test for General Relativity. International Journal of Modern Physics D, 18, 2275-2282. https://doi.org/10.1142/S0218271809015904 
[21] Matsson, L. (2016) Higgs-Like Mechansim by Confinement of Quarks in a Chemical Non-Equilibrium Model. World Journal of Mechanics, 6, 441-445. https://doi.org/10.4236/wjm.2016.611031

[22] Matsson, L. (2017) On Dark Matter Identification. World Journal of Mechanics, 7, 133-141. https://doi.org/10.4236/wjm.2017.74012

[23] Tan, A., et al. (2016) Dark Matter Results from First 98.7 Days of Data from the PandaX-II Experiment. Physical Review Letters, 117, Article ID: 121303.

[24] Akireb, D.S., et al. (2017) Results from a Search for Dark Matter in the Complete LUX Exposure. Physical Review Letters, 118, Article ID: 021303.

[25] Buckley, M.R. and DiFranzo, A. (2018) Collapsed Dark Matter Structures. Physical Review Letters, 120, Article ID: 051102. https://doi.org/10.1103/PhysRevLett.120.051102

[26] Silk, J. (2018) Molecular Ionization Rates and Ultracompact Dark Matter Minihalos. Physical Review Letters, 121, Article ID: 231105. https://doi.org/10.1103/PhysRevLett.121.231105 